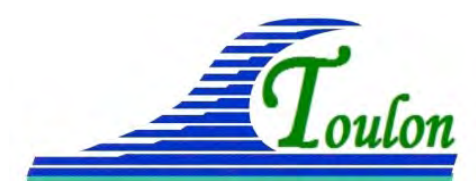

$X I V^{\text {èmes }}$ Journées Nationales Génie Côtier - Génie Civil

Toulon, 29 juin au $1^{\text {er juillet } 2016}$

DOI:10.5150/jngcgc.2016.054 @ Editions Paralia CFL

disponible en ligne - http://www.paralia.fr - available online

\title{
De l'échelle nationale à l'échelle du site, cartographie et description de la ressource en énergies marines renouvelables dans un format interopérable
}

\author{
Jean DUBRANNA ${ }^{1}$, Lionel MENARD ${ }^{1}$, Thierry RANCHIN ${ }^{1}$
}

1. MINES ParisTech, PSL Research University, Centre Observation, Impacts, Energie (O.I.E.), Rue Claude Daunesse, CS 10207, 06904 Sophia Antipolis cedex, France. jean.dubranna@mines-paristech.fr ; lionel.menard@mines-paristech.fr ; thierry.ranchin@mines-paristech.fr

\section{Résumé :}

Cet article présente une caractérisation de la ressource en Energies Marines Renouvelables (EMR) le long du littoral occidental français, sur une zone allant de la côte jusqu’à $50 \mathrm{~km}$ au large. Les caractéristiques de la ressource sont établies sous forme de cartes et/ou de tableaux de valeurs avec une résolution spatiale allant de quelques kilomètres au large à $200 \mathrm{~m}$ en zones peu profondes, soit un total d'environ 73000 points. Outre les informations de gisement de houle et de courants, des éléments permettant d'évaluer l'exploitabilité d'un site, tels que la variabilité de la ressource, les fenêtres météo saisonnières, les tableaux de probabilités conjointes hauteur/période de la houle, etc, sont établis.

Les informations générées sont renseignées grâce à une Infrastructure de Données Spatiales, puis mises en libre accès au moyen de services standard interopérables via un serveur d'application THREDDS Data Server. Elles sont enfin référencées dans un catalogue de données géo-spatiales interopérable aux standard "Catalog Service for the Web". L'ensemble de ces services permet une exploration et un téléchargement aisés des informations, et assure une très haute capacité d'utilisation par des logiciels tiers.

Ce travail se destine à l'ensemble des acteurs concernés par les caractéristiques de la ressource en EMR.

Mots-clés : Energies marines, Energies renouvelables, Ressource, Etats de mer, Données ouvertes, Interopérabilité.

\section{Introduction}

Une caractérisation appropriée de la ressource pour les énergies marines renouvelables, que nous présenterons comme une description aussi détaillée que possible des contraintes hydrodynamiques venant impacter l'exploitabilité d'un site, est un des éléments identifiés comme favorisant l'émergence de cette filière. La nature et le niveau de détail des informations nécessaires dépendent de l'état d'avancement du projet en EMR et du profil de l'utilisateur de cette donnée. Plus le projet est dans un état avancé, plus les informations fournies devront être variées, précises et spatialement spécifiques. 


\section{Thème 5 - Énergies et ressources marines}

La caractérisation de la ressource en EMR au niveau français est sporadique et s'attache généralement à en décrire le gisement. Dans le cadre du projet national EMACOP, (DUFOUR et al., 2013) exposent les caractéristiques de la ressource houlomotrice pour une vingtaine de sites en Manche et Atlantique à partir de la base de données ANEMOC. (MATTAROLO et al., 2009 ; GONÇALVES et al., 2014) étudient le potentiel houlomoteur sur une zone sud-Morbihan/Loire Atlantique/Vendée, sur la base du gisement établi par un modèle numérique de simulation des états de mer sur un maillage régulier. (GUILLOU \& CHAPALAIN, 2015) exposent les caractéristiques du gisement de houle en Mer d'Iroise en s'appuyant sur une simulation rétrospective (hindcast) du champ de vagues sur un maillage non structuré. Ils y mettent en avant la forte variabilité saisonnière, interannuelle et spatiale de cette ressource. Cette zone est également couverte par (SMITH \& MAISONDIEU, 2014) qui analysent la ressource houlomotrice et hydrocinétique : une analyse complète et détaillée des caractéristiques du champ de vagues et de courants est établie sur la base d'un hindcast de 19 années, sur maillage non structuré, et à très haute résolution spatiale à la côte. Les travaux de (SMITH \& MAISONDIEU, 2014) peuvent, en toute vraisemblance, être considérés comme des travaux de référence en termes de nature des informations nécessaires pour une caractérisation pertinente de la ressource en EMR. Ils s'appuient sur les recommandations émises à l'issue du projet EquiMar (INGRAM et al., 2011). Cependant, le détail de cette caractérisation est limité à 2 points géographiques.

Les travaux présentés dans ce document ont été menés dans le cadre du projet IREMARE supporté par l'ADEME. L'ambition finale d'IREMARE est de proposer aux acteurs des EMR une source d'informations pertinentes sur les caractéristiques metocéaniques liées à leur domaine, de l'échelle nationale à l'échelle du site, pour les stades successifs de développement de leur projet. Cette ambition passe en premier lieu par la génération des informations, puis par leur diffusion au moyen de services standards interopérables. Ces 2 étapes sont présentées dans les paragraphes respectivement 2 et 3, puis 4 et 5 de ce document. Le paragraphe 6 illustre les capacités de cette nouvelle base de données qui couvre la façade maritime occidentale française depuis la côte jusqu'à $50 \mathrm{~km}$ au large. Notre étude se distingue des travaux précités par la nature des informations générées en s’appuyant en partie sur les travaux de SMITH \& MAISONDIEU (2014) et du projet EquiMar, par la couverture géographique de la zone d'étude, par le nombre de points géographiques analysés, et enfin, par la mise à disposition sans restriction des résultats de ces analyses.

\section{Données source}

\subsection{Choix de la base de données}

A notre connaissance, les simulations rétrospectives d'états de mer permettant d'établir les caractéristiques de la ressource en EMR le long du littoral français sont : BOBWA- 


\section{XIV ${ }^{\text {èmes }}$ Journées Nationales Génie Côtier - Génie Civil \\ Toulon, 29 juin au $1^{\text {er }}$ juillet 2016}

H (CHARLES et al., 2012), ANEMOC-2 (LAUGEL et al., 2014) et HOMERE (BOUDIERE et al., 2013). (DUBRANNA et al., 2015) ont identifié HOMERE comme étant la base de données la plus pertinente pour réaliser une analyse appropriée des conditions hydrodynamiques le long du littoral occidental français en vue d'une application aux EMR. (PERIGNON \& CROM, 2015) estiment également qu'HOMERE, dont les simulations ont été réalisées à l'aide du code de calcul WAVEWATCH III dans sa version 4.09, est la base de données d'états de mer la plus aboutie actuellement. Elle se distingue en particulier (i) par la durée de ses séries temporelles (19 ans, pas de temps horaire), (ii) la haute résolution de son maillage en particulier en zone côtière (200 m), (iii) la prise en compte des phénomènes physiques majeurs (interactions houle/courants, dissipation par déferlement/moutonnement /frottements au fond, variations horaires du niveau d'eau, etc.), (iv) la pertinence de ses paramètres de sortie pour une application aux EMR. Bien que HOMERE fournisse les séries temporelles du spectre omnidirectionnel de la houle discrétisé en 32 fréquences en chacun des points de son maillage, seuls les paramètres globaux ont été utilisés dans le cadre de cette étude. HOMERE est mise à la disposition des utilisateurs par Ifremer, sur demande faite par l'intermédiaire du portail Previmer (www.previmer.org). L'ensemble des informations produites dans le cadre du projet IREMARE l'ont été par l'unique exploitation/analyse de cette base de données.

\subsection{Formatage des données}

Les fichiers de HOMERE se présentent sous la forme de 228 (19 ans x 12 mois) fichiers mensuels renfermant les séries temporelles de l'ensemble des paramètres globaux (tels que la hauteur significative des vagues $H_{m 0}$, la fréquence pic $f_{p}$ ) pour la totalité des points de maillage (environ 110 000) du modèle numérique.

La mise en forme de ces fichiers a suivi la chaine d'opérations suivante :

a) sélection des paramètres globaux pertinents (10 sur un total de 37, possibilité d'en sélectionner de nouveaux),

b) pour chaque paramètre global, génération d’un fichier unique renfermant les séries temporelles de la totalité des points de maillage pour l'ensemble de la durée de la simulation. Les fichiers formatés sont enregistrés au format netCDF.

Cette mise en forme permet de faciliter le traitement systématique de la base de données. Seuls les points de maillage situés à moins de $50 \mathrm{~km}$ des côtes ont été retenus (figure 1), soit un total d'environ 73000 points.

\section{Caractérisation de la ressource}

\subsection{Choix des paramètres de caractérisation}

Suite aux consultations effectuées auprès de nombreux acteurs de la filière dans le cadre du projet IREMARE et étant données les recommandations du projet EquiMar 
(INGRAM et al., 2011), deux ensembles d'informations ont été générés ou sont en cours de génération.

Le premier ensemble est principalement destiné à la cartographie, c'est-à-dire qu'à chaque point de maillage est associée une valeur unique, comme la hauteur significative moyenne des vagues, la vitesse moyenne du courant, etc. Nous nommerons ce genre d'élément "Information cartographiable". Le deuxième ensemble d'informations ne peut être cartographié et correspond à des informations tabulaires qui sont spécifiques à chaque point du maillage. Des éléments tels que les statistiques des fenêtres météo ou les tableaux de probabilités conjointes d'un couple hauteur/période des vagues font partie de ce deuxième ensemble d'informations. Il s'agit d'"Information tabulaire" (figure 2). Le détail des informations produites ou en cours de production sur l'ensemble de la zone traitée par le projet IREMARE est présenté dans le tableau 1.

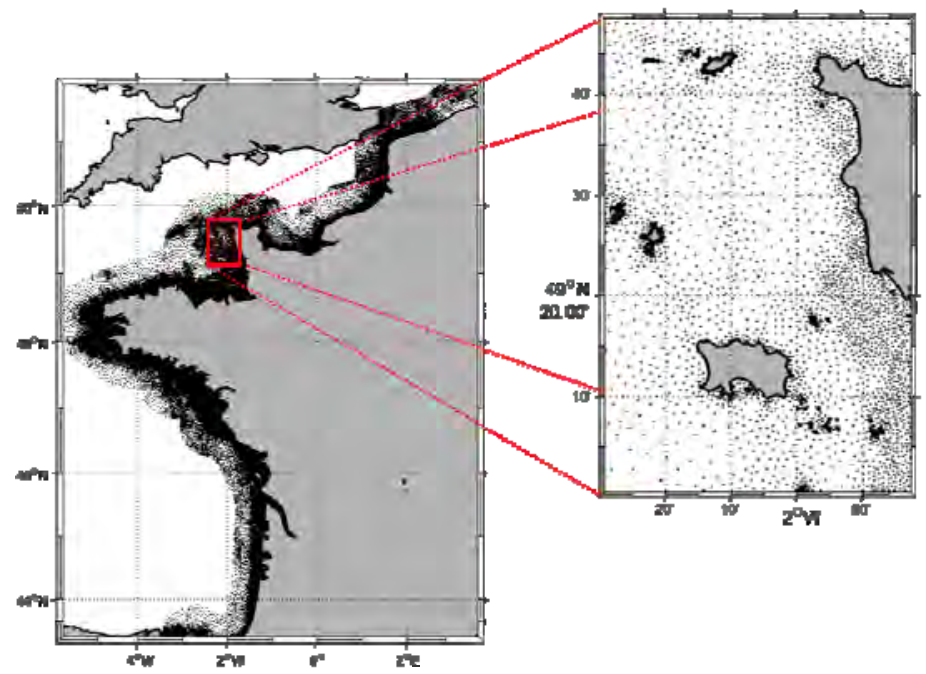

Figure 1. Emprise et détail du maillage du domaine d'étude.

\subsection{Validité des informations produites}

L'évaluation des incertitudes des informations produites, bien que fortement recommandée, ne sera pas abordée dans ce document. Nous nous appuyons sur le fait qu'HOMERE a fait l'objet de nombreuses phases de calibration et tests de validation par rapport à des mesures in situ et satellites (8 altimètres satellitaires différents), et par rapport au modèle de la National Oceanic and Atmospheric Administration (NOAA). Les coefficients de corrélation entre $\mathrm{H}_{\mathrm{m} 0}$ mesurée par des bouées à 15 sites différents et $\mathrm{H}_{\mathrm{m} 0}$ donnée par HOMERE varient entre 0,89 et 0,97, avec une légère sous-estimation par HOMERE des valeurs les plus élevées de $\mathrm{H}_{\mathrm{m} 0}$. Lorsqu'elles sont comparées à des mesures altimétriques, les coefficients de corrélation atteignent au moins 0,97. Ils valent entre 0,94 et 0,97 lorsque les valeurs de $\mathrm{H}_{\mathrm{m} 0}$ sont comparées aux sorties du modèle de la NOAA dans des eaux profondes à intermédiaires. (C. Maisondieu, communication personnelle, 17 Février 2016) recommande cependant de faire preuve de discernement 


\section{XIV èmes Journées Nationales Génie Côtier - Génie Civil \\ Toulon, 29 juin au $1^{\text {er }}$ juillet 2016}

lors de l'utilisation des données HOMERE dans les zones de profondeur limitée pour lesquelles aucune source de validation directe n'était disponible. Des détails supplémentaires concernant la validation de HOMERE sont disponibles dans (BOUDIERE et al., 2013) et attestent de la précision des variables calculées.

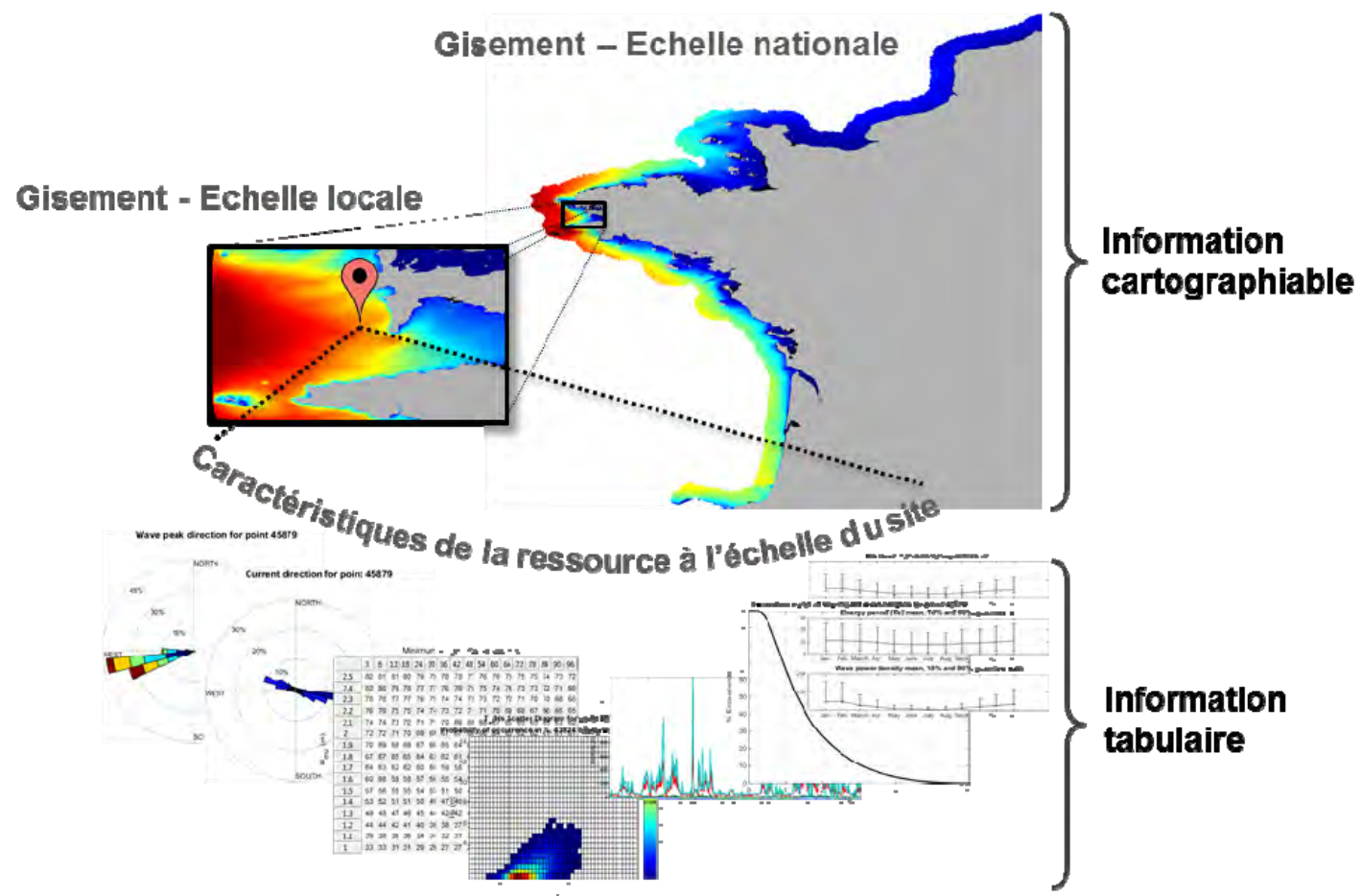

Figure 2. Illustration des informations cartographiables et informations tabulaires.

Tableau 1. Informations disponibles le 16/05/2016 et informations à venir.

\begin{tabular}{|c|c|c|}
\hline $\begin{array}{l}\text { Vagues (Informations } \\
\text { cartographiables) }\end{array}$ & $\begin{array}{l}\text { Courants (Informations } \\
\text { cartographiables) }\end{array}$ & $\begin{array}{l}\text { Données techniques } \\
\text { (Informations tabulaires) }\end{array}$ \\
\hline $\begin{array}{l}\text { Densité de puissance moyenne et } \\
\text { saisonnière des vagues, } \\
\text { Hauteur significative moyenne, } \\
\text { saisonnière et maximale (19 ans) des } \\
\text { vagues, } \\
\text { Période d'énergie et de zero- } \\
\text { crossing des vagues. } \\
\text { Informations à venir } \\
\text { Période pic des vagues. }\end{array}$ & $\begin{array}{l}\text { Vitesses maximales et moyennes du } \\
\text { courant. } \\
\text { Informations à venir } \\
\text { Puissance du courant. }\end{array}$ & $\begin{array}{l}\text { Points de maillage de Homere, } \\
\text { Probabilités conjointes } H_{m 0} / T_{e}, \\
\text { Probabilités conjointes } H_{m 0} / T_{p}, \\
\text { Densité de probabilité et fonction de } \\
\text { répartition de } H_{m 0} \text { et de la vitesse du } \\
\text { courant, } \\
\text { Roses directionnelles de la houle et } \\
\text { du courant, } \\
\text { Statistiques des fenêtres météo. }\end{array}$ \\
\hline
\end{tabular}

\section{Diffusion des données}

\subsection{Données ouvertes}

Les informations générées dans le cadre d’IREMARE sont mise en libre accès via une Infrastructure de Données Spatiales (IDS) développée par le Centre Observation, 


\section{Thème 5 - Énergies et ressources marines}

Impacts, Energie (O.I.E.) (http://www.webservice-energy.org/resources-list/iremare). Ce principe a été choisi afin de permettre aux usagers d'expérimenter leurs propres scénarios d'utilisation/combinaison des informations sans restriction de budget, et de favoriser les usages non-anticipés des informations produites.

\subsection{Services standards et interopérables}

Les informations sur la ressource sont stockées au format netCDF en respectant les Climate and Forecast Conventions (http://cfconventions.org/) en termes d'écriture des valeurs numériques et des métadonnées associées à chaque fichier. Ceci permet une exploitation optimale des fonctionnalités du serveur d'applications THREDDS Data Server qui expose le contenu des fichiers netCDF en une série de services interopérables. En particulier, ces fichiers sont accessibles aux formats standards de l'OGC (Open Geospatial Consortium) tels que Web Map Service (WMS), Web Coverage Service (WCS) et via le Data Access Protocol (DAP). Les informations sont également référencées sous forme de métadonnées dans un catalogue de données géospatiales interopérable aux standards CSW (Catalog Service for the Web) de l'OGC. L'ensemble de ces services permet une exploration et un téléchargement aisés des informations disponibles, et assure une très haute capacité de diffusion et d'utilisation par des logiciels/clients tiers. Ces informations et services sont accessibles via le lien : http://tds.webservice-energy.org/. Le catalogue de métadonnées est accessible via le lien : http://geocatalog.webservice-energy.org

Par exemple, les informations cartographiables sont stockées dans un fichier netCDF incluant les métadonnées associées à ce type d’information (résumé, unités, emprise géographique, contact, etc.). A partir du fichier netCDF, le serveur THREDDS génère un WMS directement reconnu par des clients/applications Web et/ou desktop de type SIG (Système d'Informations Géographiques) tiers tels que Géoportail, Sextant d'Ifremer, ou ArcGIS, QGIS, etc. De façon équivalente, le service OPeNDAP permet une importation puis une manipulation des valeurs numériques via des logiciels ou langages de programmation tels qu’Excel $\odot$, Matlab $\odot$, R $\odot$, IDL $\odot$, Python $\odot$, etc.

\section{Exemples d'utilisation}

\subsection{Climat de houle dans le futur parc éolien de Saint-Nazaire}

Ce premier exemple illustre l'utilisation des informations IREMARE pour une meilleure connaissance des conditions de houle au sein du futur parc éolien de SaintNazaire. Dans un premier temps, la position des parcs éoliens offshores est importée du site www.geolittoral.developpement-durable.gouv.fr au format shapefile. La carte des hauteurs significatives maximales de la houle et la position des points de maillage de HOMERE, mis à disposition dans le cadre d'IREMARE, sont ensuite importés au format WMS via le serveur THREDDS du Centre O.I.E (http://tds.webservice- 


\section{XIV ${ }^{\text {èmes }}$ Journées Nationales Génie Côtier - Génie Civil \\ Toulon, 29 juin au $1^{\text {er }}$ juillet 2016}

energy.org). Ces formats étant interopérables, l'ensemble est directement affichable, sans pré-traitement, dans un SIG, par exemple QGIS (figure 3).
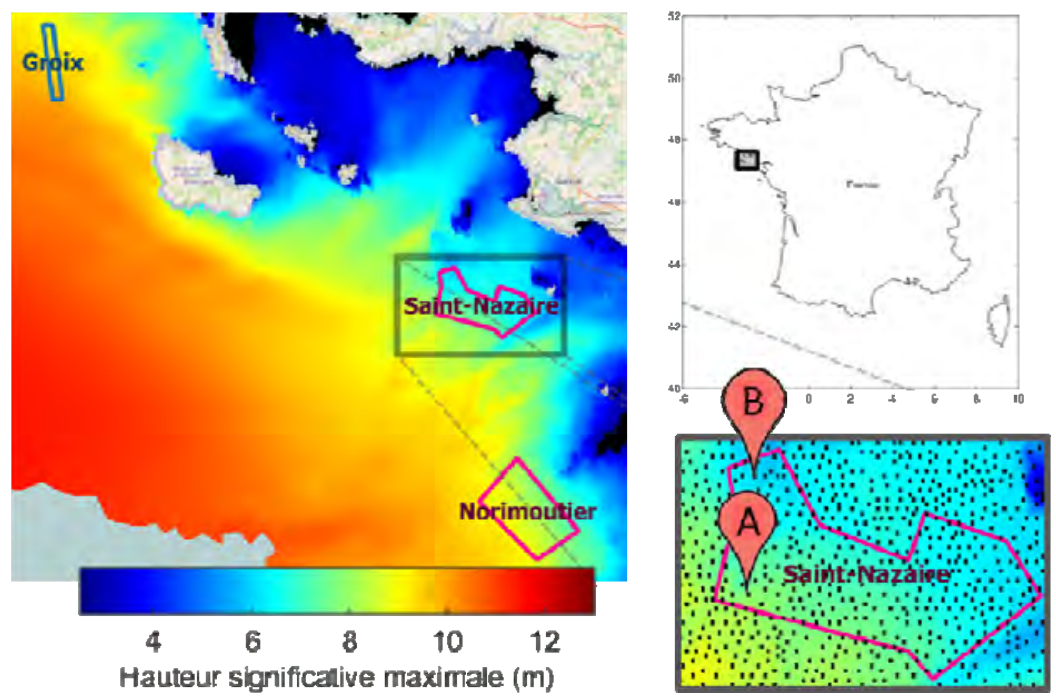

Figure 3. Affichage dans le logiciel QGIS i) des zones retenues par les appels à projet éolien offshore, ii) des hauteurs significatives maximales obtenues sur la période de simulation de HOMERE, iii) des points de grille de HOMERE (détail de Saint-Nazaire).

La carte de couleurs met en évidence des gradients de conditions de houle au sein du site éolien de Saint-Nazaire, en l'occurrence des hauteurs significatives maximales comprises entre 8,3 et 9,1 m. Ces gradients peuvent être étudiés en détail, en faisant appel à un logiciel de traitement de données tiers, grâce aux informations tabulaires qui sont mises à disposition en chaque point de maillage de HOMERE via le serveur THREDDS du Centre O.I.E. En particulier, les tableaux de probabilité d'occurrence conjointe (Hs, Tp) (figure 4) montrent une proportion plus grande de houles dont $\mathrm{H}_{\mathrm{m} 0}$ dépasse $1 \mathrm{~m}$, avec une période pic comprise entre 9 et $10 \mathrm{~s}$ au point repéré par la balise A en figure 3 qu'au point B. De même, le contenu des fenêtres météo (figure 4) renseigne sur la durée d'attente médiane entre 2 états de mer successifs identiques, à savoir dont la hauteur significative de la houle reste inférieure à une valeur donnée (entêtes de colonnes) pendant une durée minimale donnée (en-têtes de lignes). Cette durée minimale correspond également à la durée maximale requise pour une opération en mer. Par exemple, la durée médiane d'attente entre 2 fenêtres météo permettant un temps d'intervention de $12 \mathrm{~h}$ avec des $\mathrm{H}_{\mathrm{m} 0}$ inférieures à $1,2 \mathrm{~m}$ est de $90 \mathrm{~h}$ au point $\mathrm{A}$ et $76 \mathrm{~h}$ au point B. On note également que, pour des opérations d'une durée limitée à 6 h, l'usage d'un navire permettant de supporter des $\mathrm{H}_{\mathrm{m} 0}$ de $1.1 \mathrm{~m}$ au lieu de $1 \mathrm{~m}$ réduirait le temps médian d'attente de 106 h à 66 h au point $\mathrm{A}$, au bénéfice de l'opérateur. 

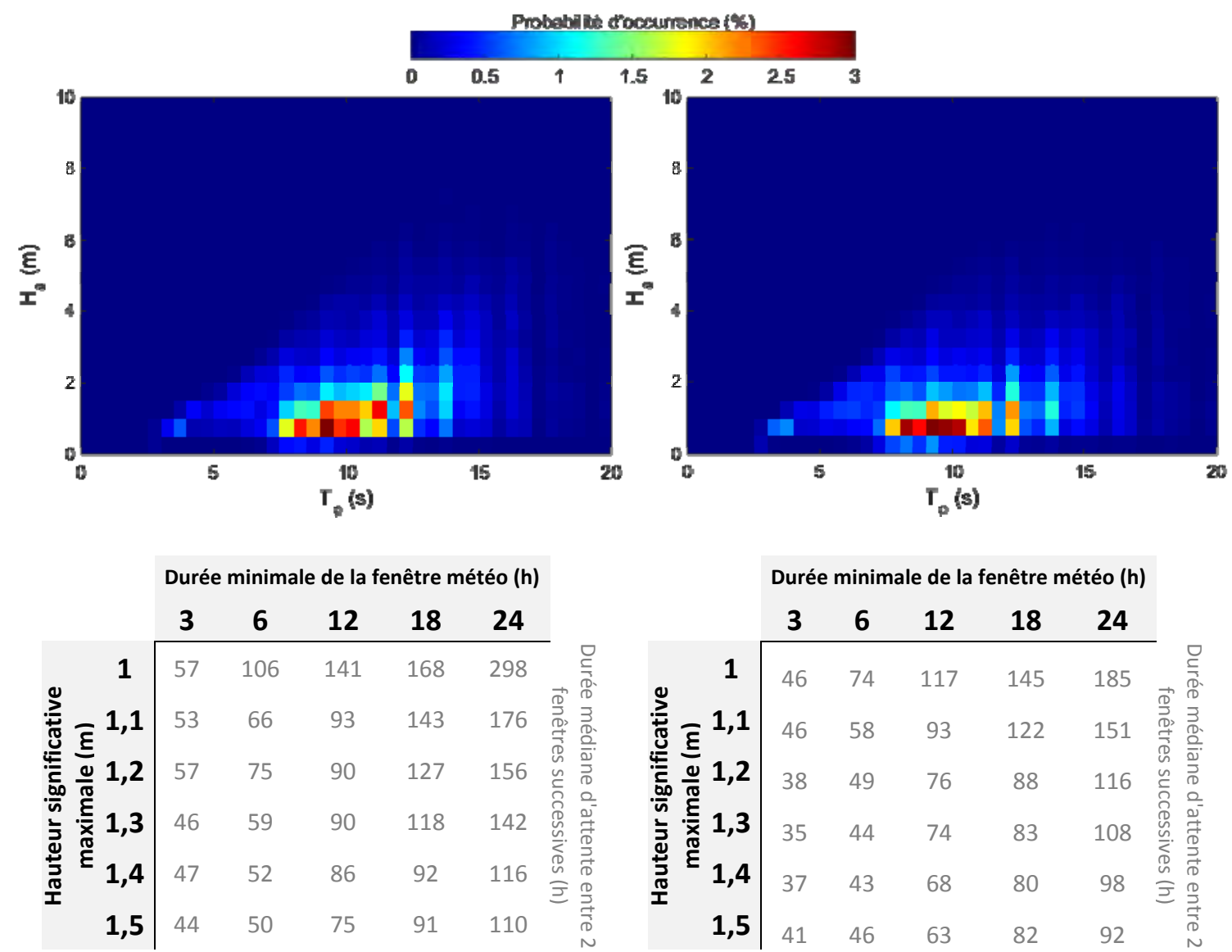

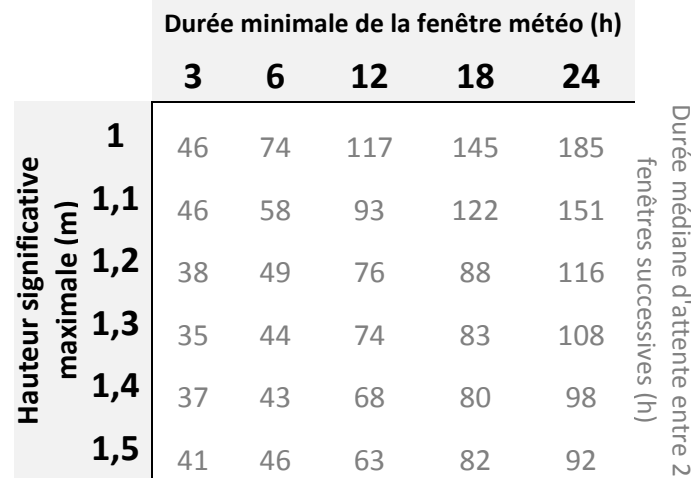

Figure 4. Probabilités conjointes (Hs, Tp) et caractéristiques des fenêtres météo.

Point A panneau de gauche, point B panneau de droite.

\subsection{Analyse multicritères}

Cet exemple vise la mise en évidence de sites répondant à des contraintes hydrodynamiques arbitraires. Ces résultats ont été obtenus uniquement par lignes de commandes effectuées à partir du logiciel Matlab $\odot$, en faisant exclusivement appel aux informations issues d'IREMARE et mises en libre accès par l'intermédiaire du serveur THREDDS. Les contraintes sont les suivantes :

a) Le site est situé à moins de $10 \mathrm{~km}$ de la côte/d'une île,

b) le site présente une densité de puissance annuelle moyenne de la houle atteinte par seulement $10 \%$ des sites localisés dans la zone décrite en a),

c) la hauteur significative maximale de la houle issue de la base de données HOMERE doit être inférieure à $10 \mathrm{~m}$,

d) la durée médiane entre 2 fenêtres météo ayant i) des conditions de hauteur significative de la houle inférieures à $1 \mathrm{~m}$, ii) permettant une intervention d'une durée maximale de 12 h (zones bleus) ou 6 h (zones bleus/rouges) ne doit pas dépasser 1 semaine en hiver. 


\section{XIV ${ }^{\text {èmes }}$ Journées Nationales Génie Côtier - Génie Civil \\ Toulon, 29 juin au $1^{\text {er }}$ juillet 2016}

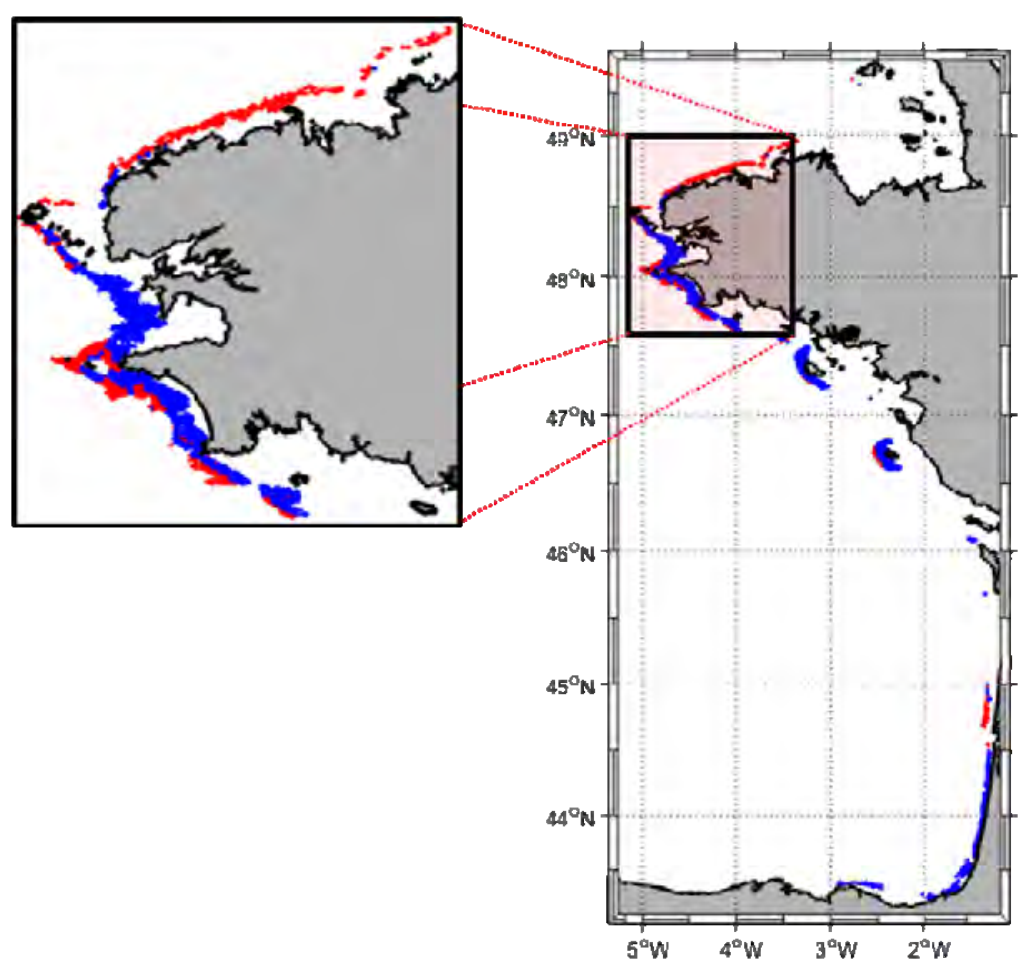

Figure 5. Résultat de l'analyse multicritères.

La figure 5 met en évidence que les sites répondant aux critères demandés se situent en Bretagne, côte aquitaine et au large de l'île d'Yeu. Une stratégie de maintenance limitant à 6 h la durée maximale d'intervention sur site (zones bleus/rouges) permettrait de retenir également des sites situés sur la côte nord-ouest de la Bretagne et la bande côtière au nord du Bassin d'Arcachon.

\section{Conclusion}

Les travaux menés dans le cadre du projet IREMARE s'attellent à la caractérisation de la ressource en Energies Marines Renouvelables par l'alimentation de la base de connaissances actuelles sur la climatologie des états de mer le long de la façade maritime occidentale française. L'ensemble des informations générées et diffusées a été élaboré grâce à l'exploitation de la base de données HOMERE (BOUDIERE et al., 2013) d'Ifremer. La mise à disposition de ces informations selon les principes de données ouvertes et d'interopérabilité permet une exploration et un téléchargement aisés des informations disponibles, et assure une très haute capacité de diffusion et d'utilisation par des logiciels tiers (QGis, Matlab, importation dans des clients web-GIS, etc).

Cette étude et ses résultats s'adressent à l'ensemble des acteurs concernés par la caractérisation de la ressource en Energies Marines Renouvelables. L'accès aux informations générées par IREMARE se fait via l’URL: http://www.webserviceenergy.org/resources-list/iremare/released-information-fr 
Thème 5 - Énergies et ressources marines

\section{Remerciements}

Le projet IREMARE est supporté par l'Agence de l'Environnement et de la Maîtrise de l’Energie (ADEME) dans le cadre de la Convention n¹505C0027.

\section{Références bibliographiques}

BOUDIERE E., MAISONDIEU C., ARDHUIN F., ACCENSI M., PINEAUGUILLOU L., LEPESQUEUR J. (2013). A suitable metocean hindcast database for the design of Marine energy converters. International Journal of Marine Energy, Vol. 3-4, pp e40-e52. http://dx.doi.org/10.1016/j.ijome.2013.11.010

CHARLES E., IDIER D., THIEBOT J., LE COZANNET G., PEDREROS R., ARDHUIN F., PLANTON S. (2012). Present Wave Climate in the Bay of Biscay: Spatiotemporal Variability and Trends from 1958 to 2001. Journal of Climate, Vol. 25, pp 2020-2039. http://dx.doi.org/10.1175/JCLI-D-11-00086.1

DUBRANNA J., RANCHIN T., MENARD L., GSCHWIND B. (2015). Production and Dissemination of Marine Renewable Energy Resource Information. 11th European Wave and Tidal Energy Conference.

DUFOUR G., MICHARD B., COSQUER E., FERNAGU E. (2013). EMACOP Project: Assessment of Wave Energy Resource Along France's Coastlines. From Sea to Shore. Meeting the Challenges of the Sea, pp 306-315.

GONCALVES M., MARTINHO P., GUEDES SORAES C. (2014). Wave energy conditions in the western French coast. Renewable Energy, Vol. 62, pp 155-163. http://dx.doi.org/10.1016/j.renene.2013.06.028

GUILLOU N., CHAPALAIN G. (2015). Numerical modelling of nearshore wave energy resource in the Sea of Iroise. Renewable Energy, Vol. 83, pp 942-953. http://dx.doi.org/10.1016/j.renene.2015.05.021

INGRAM D., SMITH G., BITTENCOURT-FERREIRA C., SMITH H. (2011). Protocols for the Equitable Assessment of Marine Energy Converters, EquiMar.

LAUGEL A., TIBERI-WADIER A.-L., BENOIT M., MATTAROLO G. (2014). ANEMOC-2 Atlantique et Méditerranée : calibration et validation de deux nouvelles bases d'états de mer construites par simulations numériques rétrospectives sur 19792010. XIIIèmes JNGCGC, Dunkerque, pp 109-120. http://dx.doi.org/10.5150/jngcgc.2014.013

MATTAROLO G., LAFON F., BENOIT M. (2009). Wave energy resource off the French coasts: the ANEMOC database applied to the energy yield evaluation of Wave Energy Converters. Proceedings of the 8th European Wave and Tidal Energy Conference, pp 247-255.

PERIGNON Y., LE CROM I. (2015). Challenging best knowledge to real conditions on the SEMREV marine test site. 11th European Wave and Tidal Energy Conference, pp 1-10.

SMITH H., MAISONDIEU C. (2014). Best practice guidelines for wave and current resource assessment. Task 1.6 of WP3 from the MERiFIC Project, 16 p. 